\title{
¿ACCEDEN AL GRADO EN MEDICINA LOS PERFILES MÁS ADECUADOS PARA FORMAR BUENOS MÉDICOS Y MÉDICOS HUMANOS?
}

\section{Do the most appropriate profiles to train good doctors and human doctors access to Medicine Degree?}

Agustín HIDALGO BALSERA

Farmacología, Departamento de Medicina, Universidad de Oviedo. Instituto Universitario de Oncología del Principado de Asturias, Fundación Cajastur. Instituto de Investigación Sanitaria de Asturias (ISPA) (España).

Correo electrónico: hidalgo@uniovi.es

Recibido: 11 de agosto de 2021

Aceptado: 16 de agosto de 2021

Llega un nuevo curso y el sistema de selección de los estudiantes que acceden a la universidad no ha variado sustancialmente: priman las calificaciones basadas en conocimientos y no se estiman actitudes ni valores. Esto aplica también a titulaciones tan vocacionales como la medicina y otras de las denominadas Ciencias de la Salud. Parecía que la adaptación de las titulaciones al Espacio Europeo de Educación superior podría cambiar, al menos en parte, el sistema de admisión puesto que las memorias de verificación de los Grados deben establecer los criterios de admisión. Pero pocos centros disponen de mecanismos que evalúen si los aspirantes realmente cumplen los requisitos exigidos, fundamentalmente los actitudinales. En esta editorial nos preguntamos si, estando así las cosas, los estudiantes que acceden al Grado en Medicina consideran que poseen actitudes y valores propios de la profesión.

En un estudio hemos pedido a los estudiantes del Grado en Medicina que valoraran su adecuación al perfil de ingreso que exige el plan de estudios. Nuestro plan de estudios define 11 rasgos de perfil de ingreso de los cuales sólo 2 tienen que ver con aspectos humanísticos y valores de la medicina ("capacidad de relacionarse con tacto y delicadeza, así como capacidad de escuchar y manifestar comprensión a sus interlocutores», e "llusión por materializar un compromiso de servicio a la salud de la comunidad»). El resto tienen que ver con características científicas y técnicas de la profesión y de algunas competencias transversales. En las competencias humanísticas, los estudiantes se atribuyen una puntuación de 4,5 puntos sobre los 5 posibles, y 


\section{¿ACCEDEN AL GRADO EN MEDICINA LOS PERFILES MÁS ADECUADOS PARA FORMAR BUENOS MÉDICOS Y MÉDICOS HUMANOS? \\ AGUSTÍN HIDALGO BALSERA}

en la puntuación media de las 11 características del perfil de ingreso la media es de 45 puntos sobre 55 , lo que supone que el $82 \%$ de los estudiantes se consideran globalmente cualificados para formarse como médicos, si bien es preocupante que un $20 \%$ se atribuyen puntuaciones subóptimas.

En otro estudio, los estudiantes han valorado su nivel de empatía según la Escala de Empatía de Jefferson (EEJ). El valor medio obtenido fue de $113,3 \pm 1,2$ puntos (rango $56-136$, sobre una puntuación máxima de 140). Estos valores son similares a los obtenidos por otros autores en otras universidades de nuestro país y de otros ${ }^{1,2}$. Por tanto, como en el estudio anterior, los valores globales pueden considerarse adecuados, aunque un $20 \%$ de los estudiantes también alcanzan, en esta escala, un valor subóptimo, lo que les convierte en candidatos de dudosa idoneidad. Este mismo estudio establece que si bien los estudiantes alcanzan calificaciones elevadas en aspectos relacionados con el hábito compasivo, son mejorables las que miden la cualidad de ponerse en el lugar del paciente.

Estos déficits son preocupantes pues, si bien se ha propuesto que la empatía parece aumentar a lo largo de la titulación ${ }^{3}$, es difícil mantenerla a lo largo del ejercicio profesional, sin olvidar que la empatía es la base del desarrollo de otros valores como el respeto, la sinceridad, la compasión o el altruismo, considerados necesarios para la transformación de los estudiantes en profesionales humanos ${ }^{4}$. Otros autores consideran que las características que definen a un buen médico no pueden ser modificadas por la educación, pero sí pueden y deben ser cuidados a lo largo de los estudios y la actividad profesional ${ }^{5}$. Por eso es impagable la labor de la Revista de Medicina y Cine y sus colaboradores en la difusión, a través de los artículos publicados, de los valores de la medicina y de toda suerte de actitudes de profesionales sanitarios recogidas en cine, arte y literatura que contribuyen a profundizar en el lado humano de la medicina y a la formación de médicos humanos y humanistas.

En otro estudio ${ }^{6}$, pedimos a los estudiantes de tres cursos diferentes (2014-15, 2015-16 y 2017-18) que describieran las razones por las que han accedido al Grado en Medicina. Participaron 367 estudiantes (252 mujeres y 115 hombres) que aportaron informaciones tales como que no hay una única razón por la que acceden al Grado en Medicina (media 2,5 \pm $0,06)$, que los valores altruistas continúan siendo los más frecuentes motivos de acceso y que la invocación de la vocación como motivo de acceso se reduce de forma importante en comparación con estudios de sólo 10 años antes ${ }^{7,8}$. En cambio, se mantiene alrededor del $25 \%$ los que acceden por motivos intelectuales y científicos. Esto sugiere que entre nuestros estudiantes encontramos el perfil de "médico híbrido» que propugnaba Rita Levi-Montalcini ${ }^{9}$; es decir, un médico "con un pie en la clínica y en la aplicación del conocimiento y otro en la generación del mismo». Continúa diciendo Levi-Montalcini que es imprescindible que estos nuevos médicos lleguen a ser líderes de los grupos de investigación "porque lo hicimos en el pasado; fuimos investigadores y médicos». No faltan entre los estudiantes que manifiestan estas preferencias los que manifiestan que ese afán de investigar, de "conocer el fascinante mundo de la biología y la armonía del funcionamiento del cuerpo" es para "adquirir nuevo conocimiento que me permita ayudar a los demás».

En todo caso, un análisis cualitativo de los motivos de acceso nos revela un porcentaje elevado de estudiantes que dicen acceder guiados por valores de la medicina tales como consolar, confortar, dar seguridad $y$, en definitiva, ayudar. Otros manifiestan su altruismo en el deseo de colaborar con Organizaciones No Gubernamentales en países en desarrollo. No faltan, por último, los que ponen el énfasis en el humanismo médico, en el viejo dicho de 


\section{¿ACCEDEN AL GRADO EN MEDICINA LOS PERFILES MÁS ADECUADOS PARA FORMAR BUENOS MÉDICOS Y MÉDICOS HUMANOS? AGUSTÍN HIDALGO BALSERA}

que la medicina es la más humana de las ciencias y la más científica de las humanidades. Un estudiante dice que la medicina le permite «combinar mi pasión humanística con la ayuda a personas, para aportar al paciente la escucha, la comprensión, y la ayuda que necesita»; otro expresa que «mi mayor motivación para ir a la universidad era la contemplación, el aprendizaje acerca del mundo. Sin embargo, la medicina me permitía dar un uso más práctico del conocimiento aprendiendo para poder ayudar a las personas sanando sus enfermedades"

Si bien el sistema universitario de admisión de estudiantes no selecciona por cualidades humanas ni actitudinales, parece que los rasgos vocaciones que llevan a los estudiantes a las aulas de los Grados en Medicina, globalmente se adaptan a los perfiles deseados en la mayoría de los casos y alcanzan puntuaciones elevadas en las cuestiones relacionadas con competencias humanas. Preocupa no obstante ese $20 \%$ de estudiantes que tienen un bajo nivel de adecuación al perfil de ingreso descrito en el plan de estudios, así como un bajo nivel de empatía, lo que sugiere que deben implementarse tanto mecanismos de selección como de formación en humanidades médicas a lo largo de los estudios de Grado y de toda su actividad profesional. A este empeño dedica esfuerzos continuados la Revista de Medicina y Cine.

En síntesis, sigue siendo válida la respuesta del profesor Graue a la pregunta ¿Por qué ser médico hoy?: "Porque la medicina es y será la profesión más completa e interesante de todas aquellas ramas del conocimiento humano: todo lo que afecta al ser humano, todo, absolutamente todo: el entorno social, ecológico, económico, cultural, familiar, sanitaria, etc., es susceptible de ser estudiado, al menos en algunos de sus aspectos o consecuencias, por ella» ${ }^{10}$.

\section{Referencias}

1. Perales A, Sánchez E, Mendoza A, Huamani C. Vocación médica en estudiantes de medicina de una universidad peruana. An. Fac. Med. 2014; 75(1): 37-42.

2. Ferreira-Valente A, Costa P, Elorduy Hernández-Vaquero $M$, Virumbrales Cancio $M$, Costa $M$, Palés J. Psychometric properties of the spanish version of the Jefferson Scale of Empathy: making sense of the total score through a second order confirmatory factor analysis. BMC Med. Educ. 2016; 16(1):1-12.

3. Esquerda M, Yuguero O, Iñas J, Pifarré J. La empatía médica, ¿nace o se hace? Evolución de la empatía en estudiantes de medicina. Aten. Primaria. 2016; 48(1): 8-14.

4. Camps V. Los valores éticos de la profesión sanitaria. Educ. Med. 2015; 16(1): 3-8.

5. González-Blasco P, Delgado-Marroquín MT, Moreto G, Altisent R. Vocación y profesionalismo: reflexiones de los estudiantes catalizados por el cine de Spielberg. Educ Med. 2019; 20(4):249-255.

6. González-García M, González-Rodríguez S, Cantabrana B, Hidalgo A. Razones por las cuales los estudiantes justifican la elección del Grado en Medicina. FEM. 2020; 23(6):351-357.

7. Soria M, Guerra M, Giménez I, Escañero IF. La decisión de estudiar medicina: características. Educ. Med. 2006; 9(2):91-97.

8. Gutiérrez-Medina S, Cuenca-Gómez D, Álvarez-De Toledo O. ¿Por qué quiero ser médico? Educ. Med. 2008; 11 (Supl 1): S1-6.

9. Levi-Montalcini R. Elogio de la imperfección. BarceIona: Tusquets; 2011.

10. Graue E. Vocación en el siglo XXI. En VV.AA. ¿Por qué ser médico hoy? Buenos Aires: Libros del Zorzal; 2009. p. 215-228. 
¿ACCEDEN AL GRADO EN MEDICINA LOS PERFILES MÁS ADECUADOS PARA FORMAR BUENOS MÉDICOS Y MÉDICOS HUMANOS?

AGUSTÍN HIDALGO BALSERA

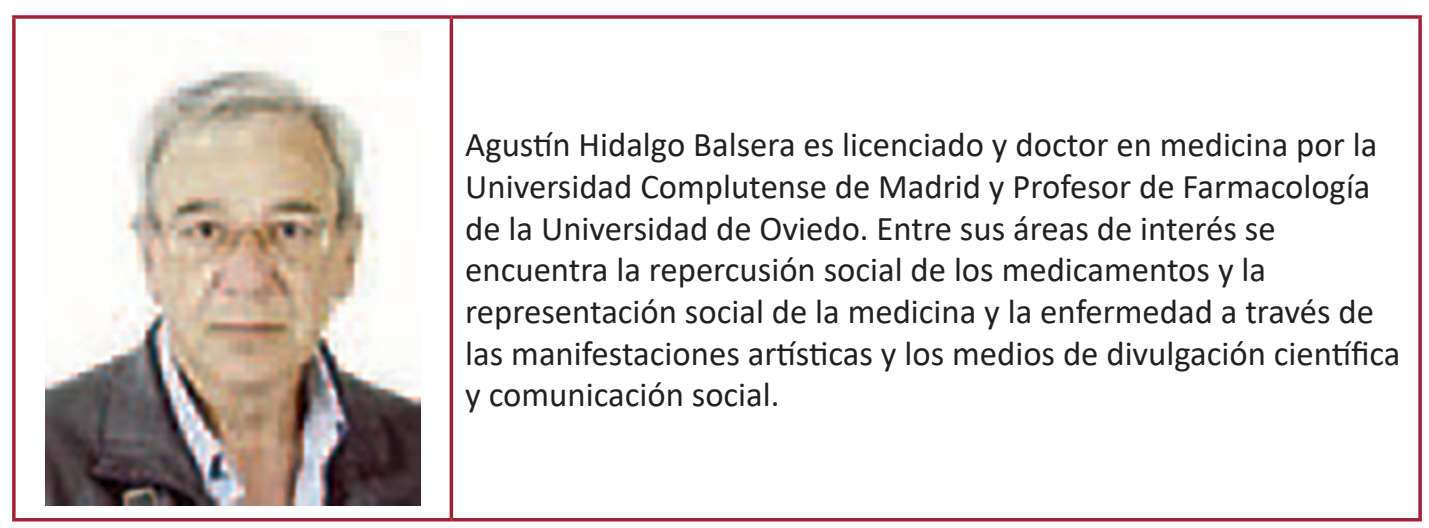

